\title{
ON STRONG FORM OF ARZELA CONVERGENCE
}

\author{
JANINA EWERT \\ Department of Mathematics \\ Pedagogical University \\ Arciszewskiego $22 \mathrm{~b}$ \\ 76-200 Slupsk, POLAND
}

(Received November 30, 1995 and in revised form April 23, 1996)

\begin{abstract}
We define some new type of convergence of nets of functions which is formulated in terms of open covers. It preserves continuity and under some assumptions implies (or coincides with) the Arzela quasi-uniform convergence. Furthermore, the introduced strong convergence is used for characterization of compactness and regularity of a topological space.
\end{abstract}

KEY WORDS AND PHRASES: Arzela convergence, almost compact space, uniform space 1991 AMS SUBJECT CLASSIFICATION CODES: 54A20.

\section{INTRODUCTION}

Let $f_{n}: X \rightarrow Y, n \geq 1$, be continuous functions and let $f: X \rightarrow Y$ be a limit of the sequence $\left\{f_{n}: n \geq 1\right\}$. Which assumptions on the convergence guarantee the continuity of $f$ ? This question led to defining various types of convergences for nets of functions with values in metric or uniform spaces $[1,2,3,4,5]$. But initial notions in this problem-continuity and pointwise convergence-are depending on topologies only and they can be considered even then if spaces are not uniformizable. This is the motivation of our paper. We define some new form of convergence formulated in terms of open covers; it preserves continuity and under some assumptions implies (or coincides with) the Arzela quasiuniform convergence.

Let $X, Y$ be topological spaces; the symbols $F(X, Y)$ and $C(X, Y)$ are used to denote the class of all functions or all continuous functions from $X$ to $Y$, respectively. For any set $A$ its closure is denoted by $C l A$.

A net $\left\{f_{j}: j \in J\right\} \subset F(X, Y)$ is said to be strongly convergent to $f \in F(X, Y)$ if:

- this net is pointwise convergent to $f$, and

- for each open cover $\mathcal{A}$ of $Y$ and each $j_{0} \in J$ there exists a finite set $J_{0} \subset J$ such that $j \geq j_{0}$ for $j \in J_{0}$ and for each $x \in X$ there are $j \in J_{0}$ and $W \in \mathcal{A}$ with $\left\{f(x), f_{j}(x)\right\} \subset W$

THEOREM 1. Let $X$ be a topological space and $Y$ a regular one. If a net $\left\{f_{j}: j \in J\right\} \subset C(X, Y)$ is strongly convergent to a function $f: X \rightarrow Y$, then $f \in C(X, Y)$.

PROOF. Let $x_{0} \in X$ and let $W, V$ be open sets in $Y$ such that $f\left(x_{0}\right) \in V \subset C l V \subset W$, then $\mathcal{A}=\{W, Y \backslash C l V\}$ is an open cover of $Y$. Now we can choose $j_{0} \in J$ and a finite subset $J_{0} \subset J$ such that

$$
\begin{aligned}
j \geq j_{0} & \text { for } \quad j \in J_{0}, \\
f_{j}\left(x_{0}\right) \in V & \text { for } j \in J, j \geq j_{0},
\end{aligned}
$$

and for each $z \in X$ there is $j_{z} \in J_{0}$ with $\left\{f(z), f_{j_{z}}(z)\right\} \subset W$ or $\left\{f(z), f_{j_{z}}(z)\right\} \subset Y \backslash C l V$ There exists a neighborhood $U$ of $x_{0}$ such that $f_{j}(U) \subset V$ for $j \in J_{0}$, thus $f_{j}(U) \cap(Y \backslash C l V)=\emptyset$ for $j \in J_{0}$ 
Hence for each $x \in U$ we have $\left\{f(x), f_{J_{x}}(x)\right\} \subset W$ which implies $f(U) \subset V$ and the proof is completed

Now we are going to compare the strong convergence with other ones. To begin with let us observe that the uniform convergence and the strong one are independent as shown in the following.

EXAMPLES. In the space $R$ of real numbers with the usual metric let us consider functions $f_{n}, f: R \rightarrow R$ defined by $f_{n}(x)=x+\frac{1}{n}, f(x)=x$ for each $x \in R, n \geq 1$; so the sequence $\left\{f_{n}: n \geq 1\right\}$ is uniformly convergent to $f$. Now, taking open intervals $U_{n}=\left(n-\frac{1}{2 n}, n+\frac{1}{2 n}\right)$ and $V_{n}=\left(n+\frac{1}{3 n}, n+1-\frac{1}{3 n}\right)$ for $n \geq 1$ we obtain the open cover $\mathcal{A}=\left\{U_{n}, V_{n}: n \geq 1\right\} \cup\{(-\infty, 3)\}$ of $R$. For fixed $n, k$ with $k>n>1$ and for each $j$ with $n<j<k$ we have that $U_{k}$ is the unique set in $\mathcal{A}$ containing $f(k)$ and $\left|f_{\jmath}(k)-f(k)\right|=\frac{1}{\jmath}>\frac{1}{2 k}$, so $f_{\jmath}(k) \notin U_{k}$. Hence the sequence $\left\{f_{n}: n \geq 1\right\}$ is not strongly convergent to $f$.

Now let $g_{n}, g: R \rightarrow R$ be functions given by:

$$
\begin{gathered}
g(x)=0 \text { for each } x \in R, \quad \text { and } \\
g_{n}(x)= \begin{cases}\frac{1}{n}, & \text { if } x \in(-\infty, n-1] \cup[n+1, \infty) \\
\left(1-\frac{1}{n}\right) x-n+2, & \text { if } x \in[n-1, n] \\
\left(\frac{1}{n}-1\right) x+n, & \text { if } x \in[n, n+1] .\end{cases}
\end{gathered}
$$

The sequence $\left\{g_{n}: n \geq 1\right\}$ strongly converges to $g$ but not uniformly.

In a uniform space $(Y, \mathcal{V})$ for any $V \in \mathcal{V}$ and $y \in Y$ we will write $V[y]=\{z \in Y:(y, z) \in V\}$. A net $\left\{f_{j}: j \in J\right\} \subset F(X, Y)$ is said to be $\mathcal{V}$-quasi-uniformly in the sense of Arzela convergent to $f \in F(X, Y)$ if

- it is pointwise convergent to $f$, and

- for each $V \in \mathcal{V}$ and $j_{0} \in J$ there exists a finite set $J_{0} \subset J$ with $j \geq j_{0}$ for $j \in J_{0}$ and for each $x \in X$ there is $j \in J_{0}$ such that $\left(f(x), f_{j}(x)\right) \in V[1,2]$.

Let us remark that the quasi-uniform convergence is strictly connected with a uniformity (or metric) For instance let $X=Y=(0, \infty), d_{1}(x, y)=|x-y|$ and $d_{2}(x, y)=\left|x^{2}-y^{2}\right|$; then $d_{1}$ and $d_{2}$ are topologically equivalent metrics on $Y$. Putting $f_{n}(x)=x+\frac{1}{n}, f(x)=x$ for $x \in X, n \geq 1$ we have the sequence $\left\{f_{n}: n \geq 1\right\}$ which converges to $f d_{1}$-uniformly but it is not $d_{2}$-quasi-uniformly convergent to $f$.

PROPOSITION. Yet $Y$ be a completely regular space. If a net $\left\{f_{j}: j \in J\right\} \subset F(X, Y)$ is strongly convergent to a function $f: X \rightarrow Y$, then for each compatible uniformity $\mathcal{V}$ on $Y$ this net $\mathcal{V}$-quasi-uniformly converges to $f$

PROOF. Let $\mathcal{V}$ be a compatible uniformity on $Y$ and $V \in \mathcal{V}$ We take an open set $W \in \mathcal{V}$ with $W=W^{-1}$ and $W^{2} \subset V$. Then it suffices to consider the open cover $\mathcal{A}=\{W[y]: y \in Y\}$.

A uniform space $(Y, \mathcal{V})$ (or simply a uniformity $\mathcal{V}$ ), is said to have the Lebesgue property if for each open cover $\mathcal{A}$ of $Y$ there is $V \in \mathcal{V}$ such that $\{V[y]: y \in Y\}$ is a refinement of $\mathcal{A}[6]$.

It is easy to see that if $\mathcal{V}$ has the Lebesgue property, then for each $X$ the strong convergence in $F(X, Y)$ coincides with the $\mathcal{V}$-quasi-uniform one.

The Lebesgue property is closely related to the paracompactness, namely we have the following

THEOREM 2 [6]. Let $Y$ be a Tychonoff space Then $Y$ possesses a uniformity $\mathcal{V}$ compatible with the given topology for which $(Y, \mathcal{V})$ has the Lebesgue property if and only if $Y$ is paracompact. Under this condition, this uniformity is the finest of all compatible uniformities, and is unique.

So, as a simple consequence we have

COROLLARY. Let $Y$ be a paracompact space and let $\mathcal{V}$ be the finest compatible uniformity on $Y$. Then for each $X$ the strong convergence in $F(X, Y)$ is equivalent to the $\mathcal{V}$-quasi-uniform one

A topological space $X$ is called almost compact if for each open cover $\mathcal{A}$ of $X$ there is a finite number $A_{1}, A_{2}, \ldots, A_{n} \in \mathcal{A}$ such that $X=\bigcup_{i=1}^{n} C l A_{2}[7$, p. 239]. 
THEOREM 3. Let $X$ be an almost compact space, $Y$ a Tychonoff one and let $\left\{f_{\jmath}: \jmath \in J\right\} \subset$ $F(X, Y), f \in C(X, Y)$. Then the following conditions are equivalent:

(a) the net $\left\{f_{j}: j \in J\right\}$ is strongly convergent to $f$;

(b) for each compatible uniformity $\mathcal{V}$ on $Y$ the net $\left\{f_{j}: j \in J\right\}$ is $\mathcal{V}$-quasi-uniformly convergent to $f$,

(c) there exists a compatible uniformity $\mathcal{V}$ on $Y$ such that the net $\left\{f_{j}: j \in J\right\}$ is $\mathcal{V}$-quasi-uniformly convergent on $f$.

PROOF. In virtue of the proposition it suffices to show the implication (c) $\Rightarrow$ (a) Let $\mathcal{V}$ be a compatible uniformity on $Y$ such that the net $\left\{f_{j}: j \in J\right\}$ is $\mathcal{V}$-quasi-uniformly convergent to $f$ We fix an open cover $\mathcal{A}$ of $Y$ and $j_{0} \in J$. Then for each $x \in X$ we choose a set $W_{x} \in \mathcal{A}$ and $V_{x} \in \mathcal{V}$ with $V_{x}=V_{x}^{-1}$ and $V_{x}^{3}[f(x)] \subset W_{x}$ Since $f$ is continuous for each $x \in X$ there is a neighborhood $U_{x}$ of $x$ for which $f\left(U_{x}\right) \subset V_{x}[f(x)]$. From the assumption on $X$ the open cover $\left\{U_{x}: x \in X\right\}$ contains a finite family $\left\{U_{x_{1}}, U_{x_{2}}, \ldots, U_{x_{n}}\right\}$ such that $X=\bigcup_{k=1}^{n} C l U_{x_{k}}$. We put $V=\bigcap_{k=1}^{n} V_{x_{k}}$. Now, by the $\mathcal{V}$-quasi-uniform convergence there exists a finite set $J_{1} \subset J$ such that. $j \geq j_{0}$ for each $j \in J_{1}$ and for each $z \in X$ we can take $j_{z} \in J_{1}$ with $\left(f(z), f_{j_{z}}(z)\right) \in V$. Furthermore, $z \in C l U_{x_{k}}$ for some $k, k \leq n$ Hence

$$
f(z) \in f\left(C l U_{x_{k}}\right) \subset C l V_{x_{k}}\left[f\left(x_{k}\right)\right] \subset V_{x_{k}}^{2}\left[f\left(x_{k}\right)\right] \subset W_{x_{k}}
$$

and

$$
f_{j_{z}}(z) \in V[f(z)] \subset V_{x_{k}}[f(z)] \subset V_{x_{k}}^{3}\left[f\left(x_{k}\right)\right] \subset W_{x_{k}} .
$$

So we have shown that for each $z \in X$ it holds $\left\{f(z), f_{j_{z}}(z)\right\} \subset W_{x_{k}}$ for some $j_{z} \in J_{1}$ and $W_{x_{k}} \in \mathcal{A}$, which finishes the proof.

THEOREM 4. For a topological space $X$ let us consider the following properties:

(a) the strong convergence in $F(X,[0,1])$ coincides with the pointwise one;

(b) $X$ is an almost compact space;

(c) for each regular space $Y$ the strong convergence in $C(X, Y)$ is equivalent to the pointwise one; then (a) $\Rightarrow$ (b) $\Rightarrow$ (c).

PROOF. We will show the implication (a) $\Rightarrow$ (b). Let $\mathcal{A}$ be an open cover of $X$ and let $\left\{U_{s}: s \in S\right\}$ be the family of all finite sums of sets belonging to $\mathcal{A}$. We define a relation " $\leq$ " in $S$ assuming $s_{1} \leq s_{2}$ iff $U_{s_{1}} \subset U_{s_{2}}$; so $(S, \leq)$ is a directed set. Now let us consider functions $f_{s}, f: X \rightarrow[0,1], s \in S$, given by $f(x)=1$ for $x \in X$ and

$$
f_{s}(x)=\left\{\begin{array}{lll}
1, & \text { if } & x \in C l U_{s} \\
0, & \text { if } & x \in X \backslash C l U_{s}
\end{array}\right.
$$

It is easy to see that $f$ is the pointwise limit of the net $\left\{f_{s}: s \in S\right\}$; thus, by the assumption this net strongly converges to $f$. Hence for the open cover $\mathcal{G}=\left\{\left[0, \frac{1}{2}\right),\left(\frac{1}{4}, \frac{3}{4}\right),\left(\frac{1}{2}, 1\right]\right\}$ of $[0,1]$ and a fixed $s_{0} \in S$ there exists a finite set $S_{1} \subset S$ with $s \geq s_{0}$ for $s \in S_{1}$ and for each $x \in X$ some $s \in S_{1}$ can be taken such that both $f(x)$ and $f_{s}(x)$ are contained in the same set from $\mathcal{G}$. This implies that for each $x \in X$ there is $s \in S_{1}$ for which $f_{s}(x)=1$, which means $X=\bigcup\left\{C l U_{s}: s \in S_{1}\right\} \quad$ From the definition of the sets $U_{s}$ it follows that $\mathcal{A}$ contains a finite family $\mathcal{A}_{1}$ with $X=\bigcup\left\{C l U: U \in \mathcal{A}_{1}\right\}$, so $X$ is almost compact

Now, we suppose that $X$ is almost compact, $Y$ is a regular space and $\left\{f_{j}: j \in J\right\} \subset C(X, Y)$ is a net of functions which pointwise converges to some $f \in C(X, Y)$. Let $\mathcal{A}$ be an open cover of $Y$ and $j_{0} \in J \quad$ For each set $W \in \mathcal{A}$ and each point $y \in W$ we choose an open set $V_{y, W}$ with $y \in V_{y, W} \subset$ $C l V_{y, W} \subset W$ Then $\left\{V_{y, W}: W \in \mathcal{A}, y \in W\right\}$ is an open cover of $Y$, so

$$
\left\{f^{-1}\left(V_{y, W}\right) \cap f_{j}^{-1}\left(V_{y, W}\right): W \in \mathcal{A}, y \in W, j \in J, j \geq j_{0}\right\}
$$

is an open cover of $X$. Since $X$ is almost compact there exists a finite family $\left\{f^{-1}\left(V_{y_{k}, W_{k}}\right) \cap f_{j_{k}}^{-1}\left(V_{y_{k}, W_{k}}\right)\right.$ : $k=1,2, \ldots, n\}$ such that 


$$
X=\bigcup_{k=1}^{n} C l\left(f^{-1}\left(V_{y_{k}, W_{k}}\right) \cap f_{j_{k}}^{-1}\left(V_{y_{k}, W_{k}}\right)\right) .
$$

Let us put $J_{1}=\left\{j_{1}, j_{2}, \ldots, j_{n}\right\}$; then $j_{k} \geq j_{0}$ for each $j_{k} \in J_{1}$. Furthermore for each $x \in X$ there is $j_{k} \in J_{1}$ and $V_{y_{k}, W_{k}}$ for which it holds

$$
x \in C l\left(f^{-1}\left(V_{y_{k}, W_{k}}\right) \cap f_{j_{k}}^{-1}\left(V_{y_{k}, W_{k}}\right)\right) \subset f^{-1}\left(C l V_{y_{k}, W_{k}}\right) \cap f_{\jmath_{k}}^{-1}\left(C l V_{y_{k}, W_{k}}\right),
$$

thus $f(x) \in W_{k}$ and $f_{j_{k}}(x) \in W_{k}$ which finishes the proof.

Let us observe that in the above theorem the implication (a) $\Rightarrow$ (b) is not reversible; moreover (b) $\Rightarrow$ (c) is not true if in (c) the $C(X, Y)$ is replaced by $F(X, Y)$. It suffices to take into account $X=Y=[0,1]$ with the usual metric and functions $f_{n}, f:[0,1] \rightarrow[0,1], n \geq 1$, given by $f_{n}(x)=x^{n}$ for $n \geq 1, x \in[0,1]$ and $f(1)=1, f(x)=0$ if $x \in[0,1)$.

THEOREM 5. For a Tychonoff space $X$ the following conditions are equivalent:

(a) for each topological space $Y$ the strong convergence is equivalent to the pointwise one on $C(X, Y)$;

(b) the strong convergence coincides on $C(X[0,1])$ with the pointwise one;

(c) $X$ is a compact space.

PROOF. The implication (a) $\Rightarrow(b)$ is evident. Let (b) be satisfied and let $\mathcal{V}$ be the uniformity on $[0,1]$ determined by the usual metric. Since $([0,1], \mathcal{V})$ has the Lebesgue property, the $\mathcal{V}$-quasiuniform convergence coincides with the strong convergence. Thus, according to (b), the $\mathcal{V}$-quasiuniform convergence is equivalent to the pointwise one on $C(X,[0,1])$. So, from $[2, T h .6]$ it means that $X$ is compact. Finally we assume that $X$ is compact. Let $Y$ be a topological space and let $\left\{f_{j}: j \in J\right\}$ be a net in $C(X, Y)$ which pointwise converges to $f \in C(X, Y)$. Now, for an open cover $\mathcal{A}$ of $Y$ and for fixed $j_{0} \in J$ it suffices to consider the open cover $\left\{f^{-1}(W) \cap f_{j}^{-1}(W): W \in \mathcal{A}, j \in J, j \geq j_{0}\right\}$ of $X$, and the proof is completed.

THEOREM 6. Let $Y$ be a Hausdorff space. If for each topological space $X$ the strong convergence coincides with the pointwise one on $C(X, Y)$, then $Y$ is a regular space

PROOF. Assume that $Y$ is not regular and let $T$ denote the topology on $Y$. There exists an open set $U_{0}$ and a point $a \in U_{0}$ such that for each neighborhood $V$ of $a$ we have $C l v \not \subset u_{0}$; hence $C l u_{0} \neq Y$ and we cn fix a point $b \in Y \backslash C l u_{0}$. By $N(a)$ we denote the family of all neighborhoods of $a$ which are contained in $U_{0}$. For any $V_{1}, V_{2} \in N(a)$ we write $V_{1} \leq V_{2}$ iff $V_{2} \subset V_{1}$; thus $(N(a), \leq)$ is a directed set. If $V \in N(a)$, then $\emptyset \neq(C l V) \backslash U_{0}$; so for each $V \in N(a)$ we choose a point $x_{V} \in(C l V) \backslash U_{0}$. We denote

$$
A_{V}=\left\{x_{W}: W \in N(a), W \geq V, W \neq V\right\}, \quad \text { for } \quad V \in N(A)
$$

Furthermore let

$$
\tau=\{A \subset Y: a \notin A \text { or } A \text { contains some } V \in N(a)\} .
$$

Then $\tau$ is a topology on $Y, T \subset \tau$ and for each point $y \in Y, y \neq a$ we have $\{y\} \in \tau$. Now we define functions $f_{V}, f:(Y, \tau) \rightarrow(Y, T)$ by the following

$$
\begin{gathered}
f(x)=x \text { for } \quad x \in Y ; \\
f_{V}(x)=\left\{\begin{array}{lll}
b, & \text { if } & x \in A_{V} ; \\
x, & \text { if } & x \in Y \backslash A_{V} .
\end{array}\right.
\end{gathered}
$$

Evidently $f$ is continuous; we are going to show that all $f_{V}$ are continuous. Let $f_{V}$ be fixed If $x \in A_{V}$, then $x=x_{W}$ with $V \leq W \neq V$ and $f_{V}\left(x_{W}\right)=b$. For a $T$-neighborhood $G$ of $f_{V}\left(X_{W}\right), G \subset Y \backslash C l U_{0}$ we can take the $\tau$-neighborhood $\left\{x_{W}, b\right\}$ of $x_{W}$ for which $f_{V}\left(\left\{x_{W}, b\right\}\right) \subset G$, so $f_{V}$ is continuous on 
$A_{V}$. Now, let $x \in Y \backslash A_{V}, x \neq a$. If $G$ is a $T$-neighborhood of $f_{V}(x)$, then it suffices to take the $\tau$ neighborhood $\{x\}$ of $x$; thus $f_{V}$ is continuous on $Y \backslash\left(A_{V} \cup\{a\}\right)$. Finally, let $x=a$ and let $G$ be a $T$ neighborhood of $f_{V}(a)$. Then $G \cap U_{0} \in N(a)$, so $G \cap U_{0}$ is a $\tau$-neighborhood of a such that $f_{V}\left(G \cap U_{0}\right) \subset G$. Hence we have shown the continuity of all functions $f_{V}$. It is easy to see that the net $\left\{f_{V}: V \in N(a)\right\}$ pointwise converges to $f$ For each point $x \in\left(C l U_{0}\right) \backslash U_{0}$ we choose a $T$ neighborhood $U_{x}$ of $x$ such that $\{a, b\} \cap U_{x}=\emptyset$; then $\mathcal{A}=\left\{U_{0}, Y \backslash C l U_{0}\right\} \cup\left\{U_{x}: x \in\left(C l U_{0}\right) \backslash U_{0}\right\}$ is a $T$-open cover of $Y$. For a finite set $\left\{V_{1}, V_{2}, \ldots, V_{n}\right\} \subset N(a)$ with $V_{\imath} \neq V_{j}$ for $i \neq j ; i, j \leq n$ we can take $V \in N(a)$ such that $V_{\jmath} \leq V$ and $V_{\jmath} \neq V$ for $j \leq n$. Then we have'

$$
\begin{gathered}
f\left(x_{V}\right)=x_{V} \in U_{x_{V}}, \quad f\left(x_{V}\right) \notin Y \backslash C l U_{0}, \\
f_{V_{j}}\left(x_{V}\right)=b \in Y \backslash C l U_{0} \quad \text { and } \quad f_{V_{j}}\left(x_{V}\right) \notin U_{x_{V}} \quad \text { for } j \leq n .
\end{gathered}
$$

This means that the sets of the cover $\mathcal{A}$ do not contain none of sets $\left\{f_{V}\left(x_{V}\right), f\left(x_{V}\right)\right\}$ for $j \leq n$, hence the net $\left\{f_{V}: V \in N(a)\right\}$ is not strongly convergent to $f$ which finishes the proof.

\section{REFERENCES}

[1] ARZELÀ, C., Sulle serie di funzioni, Mem. R. Accad. Sci. Inst. Bologna ser. 5 (8), (1899-1900), 130-186, 701-744.

[2] BUKOVSKÁ, Z., BUKOVSKÝ, L. and EWERT, J., Quasi-uniform convergence and L-spaces, Real Anal. Exchange 18 (1992-93), 321-329.

[3] EWERT, J., Almost uniform convergence, Periodica Math. Hungarica 26 (1) (1993), 77-84

[4] POPPE, H., Compactness in function spaces with a generalized uniform structure II, Bull. Acad. Polon. Sci. Ser. Sci. Math. Astronom. Phys. 18 (1970), 567-573.

[5] PREDOI, M., Sur la convergence quasi-uniforme, Periodica Math. Hungarica 10 (1979), 31-40.

[6] KASAHARA, S. and KASAHARA, K., Note on the Lebesgue property in uniform spaces, Proc. Japan Acad. 31 (1955), 1615-1617.

[7] CSÁSZÁR, A., General Topology, Budapest, 1978. 


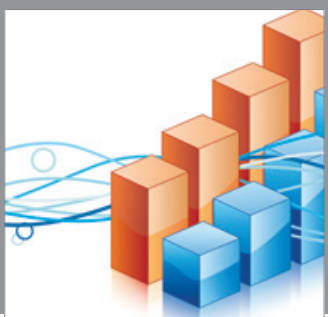

Advances in

Operations Research

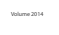

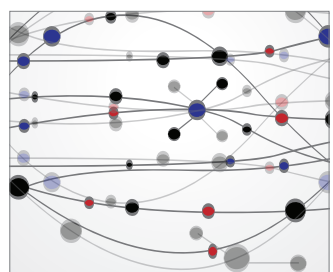

\section{The Scientific} World Journal
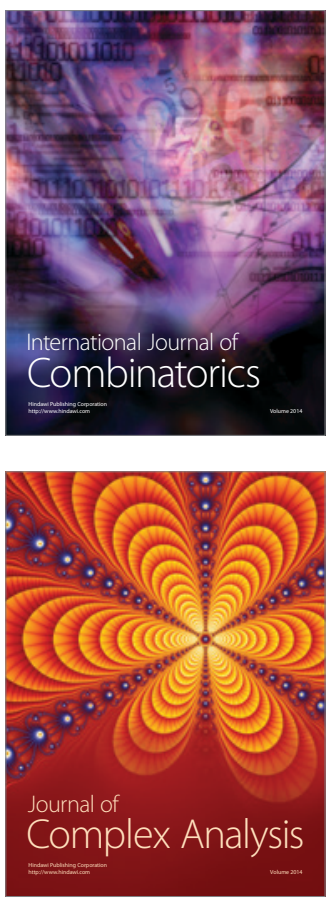

International Journal of

Mathematics and

Mathematical

Sciences
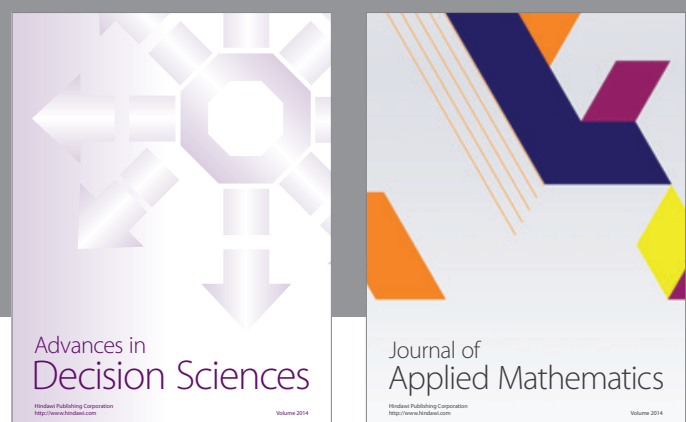

Journal of

Applied Mathematics
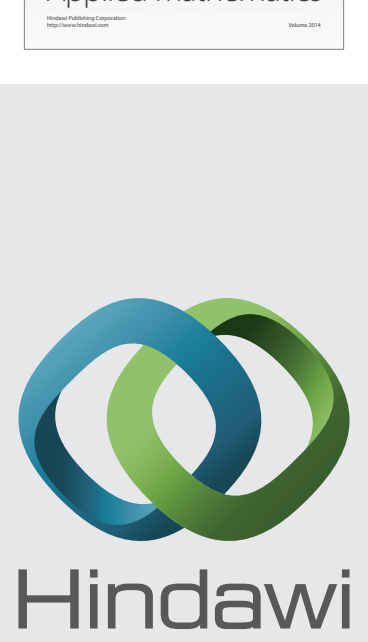

Submit your manuscripts at http://www.hindawi.com
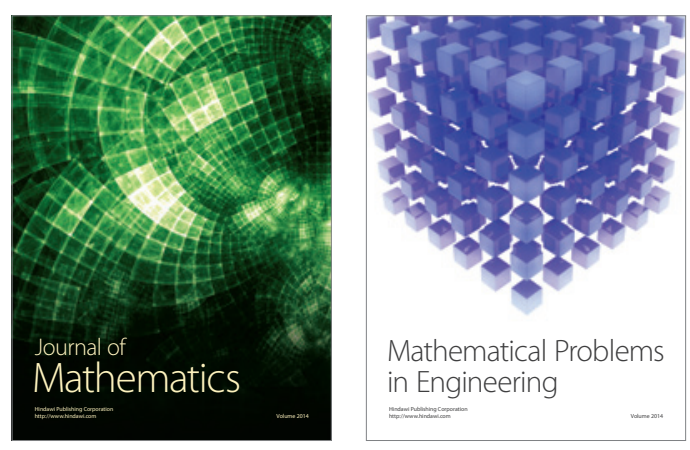

Mathematical Problems in Engineering
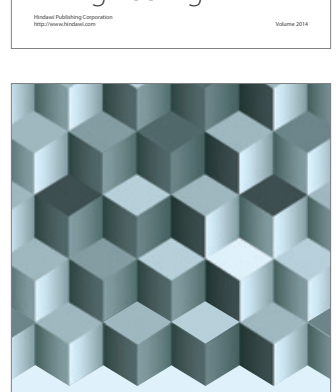

Journal of

Function Spaces
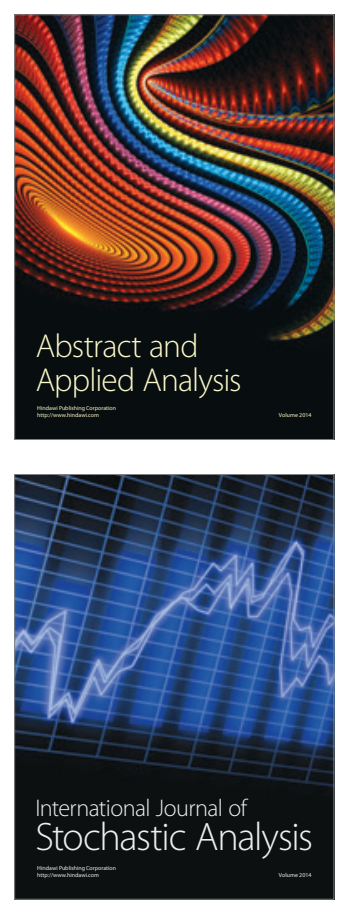

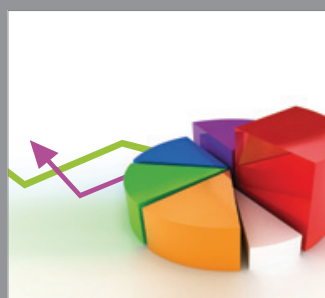

ournal of

Probability and Statistics

Promensencen
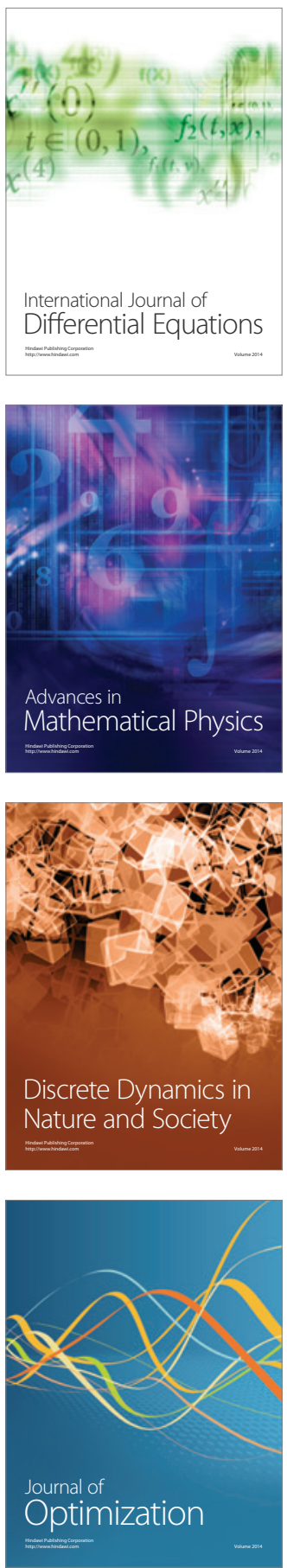